\title{
Salvage Clinical Trial Setting
}

National Cancer Institute

\section{Source}

National Cancer Institute. Salvage Clinical Trial Setting. NCI Thesaurus. Code C156600.

A clinical trial or treatment setting in which the therapy of interest is considered salvage therapy. 